\title{
Telomere-binding protein Taz1 controls global replication timing through its localization near late replication origins in fission yeast
}

\author{
Atsutoshi Tazumi, ${ }^{1}$ Masayoshi Fukuura, ${ }^{1}$ Ryuichiro Nakato, ${ }^{2}$ Ami Kishimoto, ${ }^{1}$ Tomokazu Takenaka, ${ }^{1}$ \\ Shiho Ogawa, ${ }^{1}$ Ji-hoon Song, ${ }^{1}$ Tatsuro S. Takahashi, ${ }^{1}$ Takuro Nakagawa, ${ }^{1}$ Katsuhiko Shirahige, ${ }^{2}$ \\ and Hisao Masukata ${ }^{1,3}$ \\ ${ }^{1}$ Department of Biological Sciences, Graduate School of Science, Osaka University, Toyonaka, Osaka 560-0043, Japan; ${ }^{2}$ Research \\ Center for Epigenetic Disease, Institute of Molecular and Cellular Biosciences (IMCB), University of Tokyo, Yayoi, Tokyo \\ 113-0032, Japan
}

In eukaryotes, the replication of chromosome DNA is coordinated by a replication timing program that temporally regulates the firing of individual replication origins. However, the molecular mechanism underlying the program remains elusive. Here, we report that the telomere-binding protein Taz1 plays a crucial role in the control of replication timing in fission yeast. A DNA element located proximal to a late origin in the chromosome arm represses initiation from the origin in early $S$ phase. Systematic deletion and substitution experiments demonstrated that two tandem telomeric repeats are essential for this repression. The telomeric repeats recruit Taz1, a counterpart of human TRF1 and TRF2, to the locus. Genome-wide analysis revealed that Taz1 regulates about half of chromosomal late origins, including those in subtelomeres. The Taz1-mediated mechanism prevents Dbf4-dependent kinase (DDK)-dependent Sld3 loading onto the origins. Our results demonstrate that the replication timing program in fission yeast uses the internal telomeric repeats and binding of Taz1.

[Keywords: Taz1; chromosome; initiation of replication; replication timing; telomere]

Supplemental material is available for this article.

Received April 16, 2012; revised version accepted July 24, 2012.

DNA replication is fundamental and essential for the inheritance of genetic information through generations. For complete replication of large eukaryotic chromosomes to occur within the limited period of $S$ phase, DNA replication initiates at a large number of chromosome loci known as replication origins. Replication origins do not fire simultaneously at the onset of S phase but do so at distinct time points during $S$ phase. Control of replication timing, in which replication initiates from each origin at a prefixed time during $\mathrm{S}$ phase, is common in eukaryotic chromosomes from yeast to metazoan (MacAlpine and Bell 2005; Gilbert et al. 2010). In mammalian cells, it is suggested that the replication timing program is established during a discrete time window in G1 phase, the timing decision point (TDP) (Dimitrova and Gilbert 1999). The fact that replication timings are changed coupled with alterations in histone modifications and gene expression during metazoan development

${ }^{3}$ Corresponding author

E-mail masukata@bio.sci.osaka-u.ac.jp

Article is online at http://www.genesdev.org/cgi/doi/10.1101/gad.194282.112. suggests the importance of replication timing control (RTC) (Hiratani et al. 2004, 2008; Lande-Diner et al. 2009).

The initiation of replication in eukaryotes consists of several distinct steps that are regulated by cell cycle. In G1 phase, the origin recognition complex (ORC), together with Cdc6 and Cdt1, promotes loading of a minichromosome maintenance (MCM) complex, composed of heterohexameric $\mathrm{Mcm} 2-7$, onto replication origins, resulting in a prereplicative complex (pre-RC) (Bell and Dutta 2002). Activation of two essential kinases, cyclin-dependent kinase (CDK) and Dbf4-dependent kinase (DDK), at the onset of $S$ phase promotes the assembly of several other replication factors, including Sld3, Cdc45, and GINS, leading to the unwinding of origin DNA and the initiation of DNA synthesis (Remus and Diffley 2009; Labib 2010; Tanaka and Araki 2010). In budding yeast and fission yeast, pre-RCs are formed at all of the origins, whereas specific origins are activated in early $S$ phase (Raghuraman et al. 2001; Wyrick et al. 2001; Hayashi et al. 2007). Recent studies have suggested that DDK-dependent origin loading of Sld 3 and Cdc45 is a key step in determining the timing 
of initiation (Tanaka et al. 2011). Therefore, the replication timing program seems to regulate the activation of pre$\mathrm{RCs}$ in these yeasts.

It is generally agreed that the status of chromatin has an impact on the timing of origin activation. Replication origins in euchromatin domains, where genes are actively transcribed, initiate early, while those in heterochromatin, where genes are repressed, replicate in late S phase (MacAlpine et al. 2004; Hiratani and Gilbert 2009). However, genome-wide identification of replication origins in fission yeast indicate that a subset of euchromatic origins do not fire in early S phase (Hayashi et al. 2007). Moreover, centromeric heterochromatin and the silent mating type (mat) locus replicate in early S phase (Kim et al. 2003; Hayashi et al. 2009), and interestingly, Swi6, a conserved heterochromatin protein 1 (HP1), stimulates their early replication (Hayashi et al. 2009). On the other hand, subtelomeric heterochromatin replicates very late in S phase in the presence or absence of Swi6 (Hayashi et al. 2009), suggesting that a certain mechanism independent from heterochromatin regulates replication timing. Although the checkpoint pathway represses late origins under the depletion of nucleotides by hydroxyurea $(\mathrm{HU})$ in budding yeast and fission yeast, it does not affect the replication timing of the origins (Santocanale and Diffley 1998; Santocanale et al. 1999; Hayashi et al. 2007).

In budding yeast, proximity to the telomere confers late replication of some origins (Ferguson and Fangman 1992). Telomeric DNA, consisting of TTAGGG with speciesspecific variations, becomes shorter as a result of lagging strand synthesis, and shortened telomeres are elongated by telomerase, the telomere-specific reverse transcriptase. Interestingly, long telomeres cause a delay in the replication timing of telomere-proximal origins (Bianchi and Shore 2007), suggesting the involvement of telomere DNA and probably the telomere-binding proteins in the control of replication timing in the regions proximal to telomeres. In the internal arm regions, although the importance of some unique sequence elements associating with late origins has been reported (Friedman et al. 1996; Sharma et al. 2001; Yompakdee and Huberman 2004), the molecular mechanisms underlying the control of replication timing have not been elucidated.

In the present study, we demonstrate that the telomerebinding protein Tazl plays an essential role in the control of replication timing in fission yeast. Tazl, an ortholog of mammalian TRF1 and TRF2, binds to telomeres at the ends of fission yeast chromosomes and is required for the protection and maintenance of the telomeres (Cooper et al. 1997; Kanoh and Ishikawa 2001). Although Tazl has been shown to be required for the passage of replication forks through telomeric DNA (Miller et al. 2006), the involvement of Tazl in the regulation of replication initiation in the chromosome arm regions has not been considered. Here, we show that a DNA fragment proximal to the late origin, AT2088, when inserted at ectopic sites, repressed firing from neighboring early origins in early $\mathrm{S}$ phase. The essential sequence for repression determined by deletions and substitutions resembles two tandem copies of a telomeric repeat. Interestingly, similar telomeric repeats were found near a subset of late origins. The telomeric repeats proximal to AT2088 and another late origin, AT2035, recruit Taz1 to the loci, and the recruitment of Tazl was essential for the timing control of these late origins. A 50-base-pair (bp) fragment containing two copies of the essential sequence is sufficient for the recruitment of Tazl and the timing control of a nearby origin. Remarkably, in the taz1 $1 \Delta$ strain, about half of the previously identified late origins, including those in the subtelomeres, fire in early S phase. These results indicate that Taz1, a well-defined telomere-binding protein, plays an essential role in RTC through binding to the telomeric repeats in the vicinity of internal late origins in fission yeast.

\section{Results}

Identification of the DNA element that controls replication timing

To elucidate the mechanism that regulates timing of firing at individual replication origins, we compared two replication origins, ars2004 and AT2088/ARS745, which share properties of strong replication origins but differ in replication timing. ars2004, located in the left arm of chromosome II (Fig. 1A), is a well-characterized early origin (Okuno et al. 1999; Takahashi et al. 2003) where initiation factors assemble efficiently at the onset of $S$ phase (Yamada et al. 2004; Yabuuchi et al. 2006). AT2088/ARS745 (Maundrell et al. 1988; Segurado et al. 2003 ) is a late/dormant origin located in the right arm of chromosome II (Fig. 1A). Despite the efficient formation of pre-RC at AT2088, 5-bromo-2'-deoxyuridine (BrdU), a heavy-density analog of thymidine, was not incorporated in HU-treated early S-phase cells (Hayashi et al. 2007). Both origins are located in large intergenic regions flanked by divergent promoters and contain long AT-rich regions that are essential for the replication origin activity. Moreover, they exhibit efficient ARS activity when the respective fragment is cloned on a plasmid (Supplemental Fig. S1A).

Since the previous study of early and late origins was carried out using HU-treated cells where the replication checkpoint was activated (Hayashi et al. 2007), we examined the replication timing of ars2004 and AT2088 in unperturbed $\mathrm{S}$ phase. Cells synchronously released from G2/M block by a temperature-sensitive cdc25-22 mutation were labeled for indicated periods with BrdU. The amounts of the heavy-light (HL) and light-light (LL) DNA, separated by cesium chloride $(\mathrm{CsCl})$ density gradient centrifugation, were determined by real-time PCR (qPCR) using primers amplifying ars2004 and AT2088, together with AT2024 (internal early origin control), AT2035 (late origin control), and TAS59, which is $\sim 20 \mathrm{~kb}$ distant from the right end of chromosome II (Fig. 1A). Replication kinetics showed that ars2004 and AT2024 replicated $\sim 10 \mathrm{~min}$ earlier than AT2035 and AT2088, while TAS59 replicated much later than the others (Fig. 1B). These results demonstrated that the replication timings of ars2004 and AT2088 differ in unperturbed S phase. 
Tazumi et al.

A

Chr II

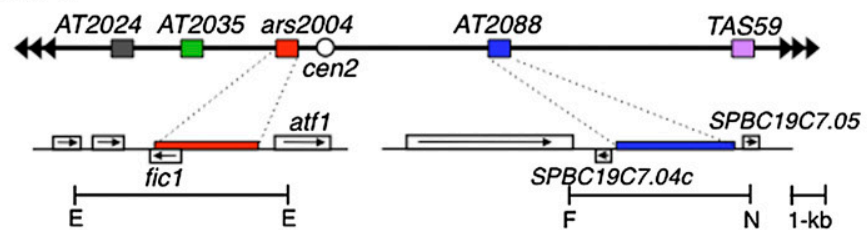

B

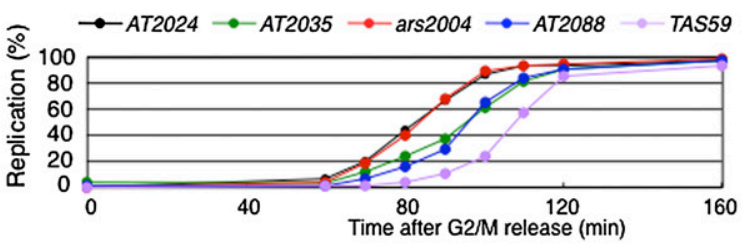

C

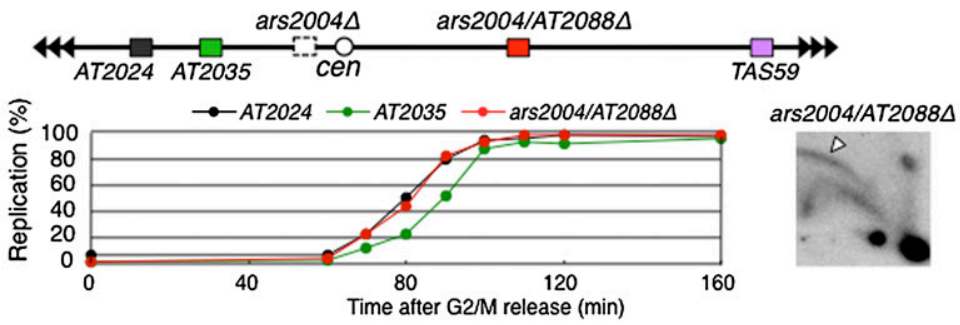

D

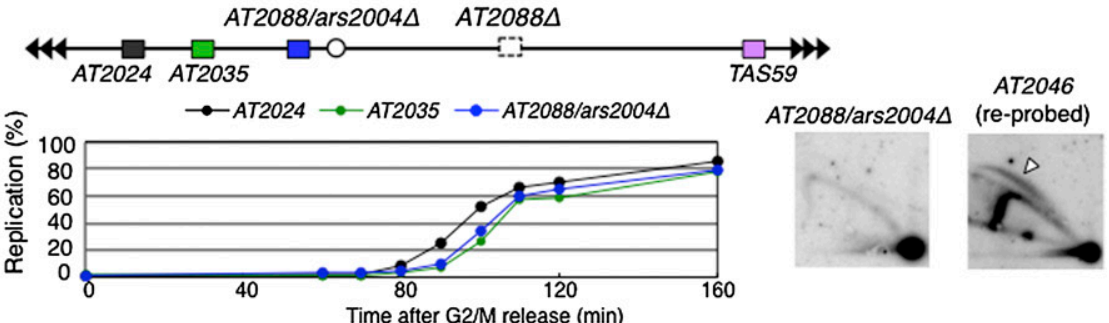

E

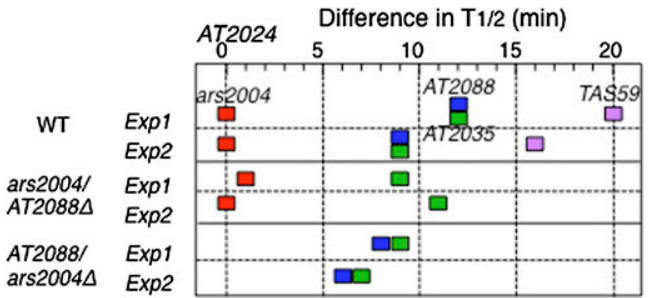

Figure 1. Replication timings of ars2004 and AT2088 origin fragments are maintained at ectopic loci. $(A)$ The locations of early and late replication origins on Schizosaccharomyces pombe chromosome II are presented schematically. The positions of early origins AT2024 (gray) and ars2004 (red); cen2; late origins AT2035 (green) and AT2088 (blue); and a subtelomeric origin, TAS59 (purple) are shown. For the ars2004 and AT2088 loci, the locations of the genes, along with the direction of transcription (arrow) and fragments (3.2-kb ars2004[red]; 3.6-kb AT2088 [blue]) used for translocation, are presented. Relevant restriction fragments (EcoT22I [E], FbaI [F], and NcoI [N]) analyzed by two-dimensional (2D) gel electrophoresis are shown below the maps. (B) Replication kinetics of early and late origins. Fission yeast $c d c 25-22 \mathrm{nmt1}-\mathrm{TK}^{+}$cells arrested at the G2/M boundary for $3 \mathrm{~h}$ at $36^{\circ} \mathrm{C}$ were released at $25^{\circ} \mathrm{C}$ in the presence of $\mathrm{BrdU}(200 \mu \mathrm{M})$. At the indicated time points, the replicated heavy-light (HL) DNA was separated from light-light (LL) DNA using cesium chloride (CsCl) density gradient centrifugation, and the amount of DNA of AT2024 (black), AT2035 (green), ars2004 (red), AT2088 (blue), and TAS59 (purple) in the LL and HL densities was determined by qPCR. The replication kinetics of each origin are presented. The results of biologically independent experiments are shown in Supplemental Figure S1B. $(C, D)$ The replication kinetics of ars2004 (red), inserted at the $A T 2088$ locus $(C)$, and AT2088, inserted at the ars2004 locus $(D)$, were obtained as described in $B$. The results of biologically independent experiments are shown in Supplemental Figure S1, C and D. The right panels show the results of 2D gel analysis of ars2004 (FbaI-NcoI fragment) at the AT2088 locus (C) and of AT2088 (EcoT22I fragment) at the ars2004 locus $(D)$ prepared at 90 min after G2/M release in the presence of $\mathrm{HU}(10 \mathrm{mM})$. (D) The membrane was rehybridized with the early origin AT2046 probe. An arrowhead indicates the bubble arc. (E) Comparison of the time required for replication in half of the cell population (T1/2). T1/2 (in minutes) for each origin was obtained from the results presented in $B-D$ and Supplemental Figure. S1B-D. The difference in T1/2 between an origin and AT2024 (early origin control) is presented. AT2035 and AT2088 replicate 6-12 min later than ars2004 and AT2024, regardless of their locations on the chromosome. 
To investigate whether the replication timings of ars2004 and AT2088 are intrinsic to the origins, the intergenic fragment containing each origin was translocated into an ectopic chromosomal context. The $3.2-\mathrm{kb}$ fragment containing ars2004 inserted at the AT2088 locus (ars2004/AT2088D) replicated as early as the control early origin AT2024 (Fig. 1C). In contrast, the AT2088 fragment inserted at the ars2004 locus (AT2088/ars2004D) replicated as late as the control late origin AT2035 (Fig. 1D). Although the absolute times of replication vary between experiments, probably due to the difference in the period required for re-entry into the cell cycle from the G2/M block, we confirmed that the difference in $\mathrm{T} 1 / 2$, the time required for replication in half of a cell population for an origin relative to that of the early origin control AT2024, was maintained (Fig. 1E). The results of two-dimensional (2D) gel electrophoresis analysis indicated that replication initiated efficiently from the ars2004 placed at the AT2088 locus, as shown by the bubble arc (Fig. 1C), and that replication was rarely initiated from the AT2088 fragment at the ars2004 locus in early S phase (Fig. 1D). Moreover, the plasmids pARS2004 and pAT2088, carrying the corresponding fragments, replicated early and late in S phase, respectively (Supplemental Fig. S1E). These results demonstrate that the replication timings are intrinsic to the ars2004 and AT2088 fragments.

If an element located in the AT2088 fragment forces the origin not to fire in early $\mathrm{S}$ phase, it might also repress another origin placed closely to the fragment. To test this possibility, the AT2088 fragment was inserted in the vicinity of ars2004 on the chromosome (Fig. 2A), and the amounts of BrdU-incorporated DNA in HU-treated early S -phase cells were analyzed for ars2004 locus and other origins. In HU-treated wild-type cells without insertion, both ars2004 and AT2024 showed robust replication, while AT2035 did not replicate efficiently (Fig. 2B). Although the insertion of the $\mathrm{ura}^{+}$fragment alone did

A

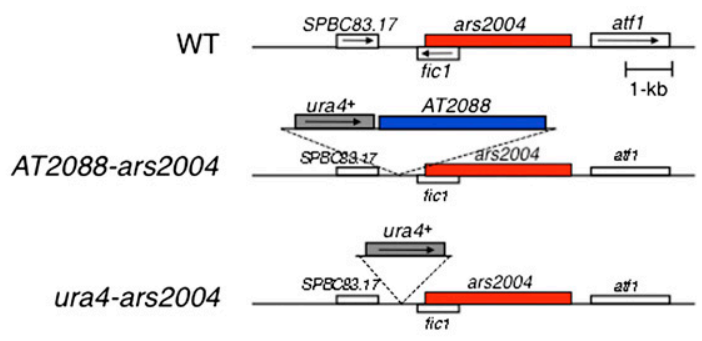

B

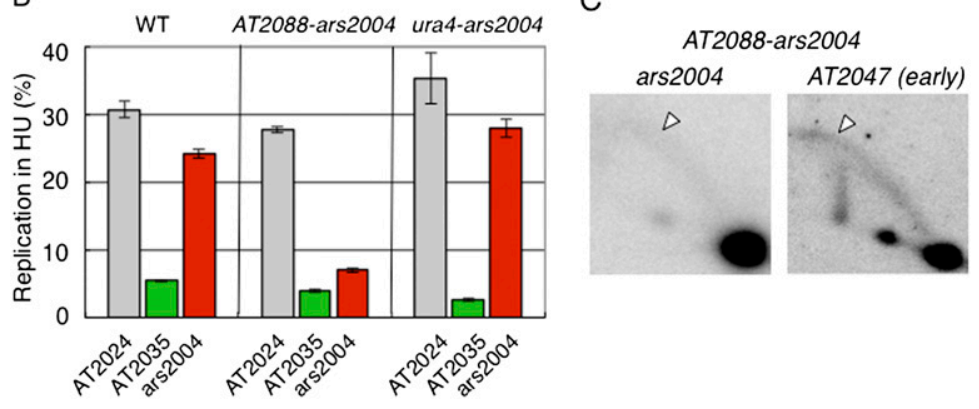

not cause a significant change (Fig. 2B), the insertion of AT2088 markedly decreased replication of ars2004, indicating that the AT2088 fragment repressed the neighboring ars2004 (Fig. 2B). This was due to a decrease in initiation from ars2004, as shown by the reduced amount of bubble arc in 2D gel analysis (Fig. 2C). Furthermore, the AT2088 fragment, when inserted at a site close to another early origin ORI12 on chromosome I (Segurado et al. 2003), also repressed the early replication of the origin (Supplemental Fig. S2B). These results showed that the AT2088 fragment has the ability to control the replication timing of proximal origins. Accordingly, we named the activity "replication timing control" (RTC) activity.

\section{Identification of the essential sequence for RTC}

To determine the minimum region of AT2088 that is required for RTC activity, AT2088 fragments lacking various regions were inserted next to ars2004 (Fig. 3A). The results presented in Figure 3B show that a 634-bp fragment (L2312) of AT2088 is sufficient for the repression of ars2004. Experiments using a series of internal deletions in the 634-bp fragment revealed that a 32-bp fragment $(2617-2648, \mathrm{~N} 2617)$ is required for the RTC and that the surrounding regions contribute to some extent (Fig. 3B). Base substitutions of every $8 \mathrm{bp}$ in the 32-bp region identified the 17-bp RTC essential sequence (from position 2624 to 2640 ) and an 8-bp important sequence (from position 2641 to 2648) (Fig. 3D).

Because RTC alters the replication timing of ectopically adjoined replication origins, its function is distinct from the replication origin activity of AT2088. The 3.6-kb intergenic fragment of AT2088 consists of two ARS fragments, each containing a highly AT-rich region (Fig. 3C; Supplemental Fig. S3B). Disruption of the RTC essential sequence, located several hundred base pairs distant from the closer AT-rich region, did not affect the

Figure 2. Repression of early initiation from the ars2004 origin by the adjoining AT2088 fragment. (A) Schematic presentation of the ars2004 locus carrying the ura4 ${ }^{+}$ -AT2088 or ura $4^{+}$insertion. (B) cdc25-22 nmt1-TK $\mathrm{K}^{+}$derivatives with or without the insertion of $\mathrm{ura}^{+}-A T 2088$ or $\mathrm{ura}^{+}$alone next to the ars2004 locus were released from the G2/M block and labeled with BrdU for $120 \mathrm{~min}$ in the presence of $\mathrm{HU}(10 \mathrm{mM})$. Replication (percentage) in the presence of $\mathrm{HU}$ was determined for ars2004 (red) as well as the control early and late origins AT2024 (gray) and AT2035 (green), respectively. The mean \pm SD obtained from multiple measurements in qPCR is presented. The results of a biologically independent experiment are presented in Supplemental Figure S2A. (C) The replication intermediates of ars2004 (StyI fragment) and a control early origin, AT2047 (StyI fragment), prepared at $90 \mathrm{~min}$ after $\mathrm{G} 2 / \mathrm{M}$ release of the HU-treated AT2088-ars2004 cells were analyzed by 2D gel electrophoresis. An arrowhead shows the bubble arc. 
Tazumi et al.

A

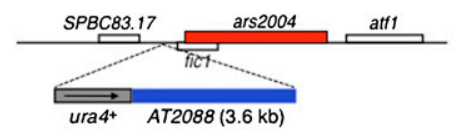

B

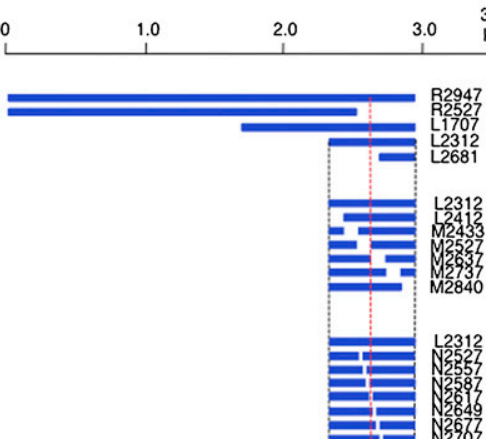

C

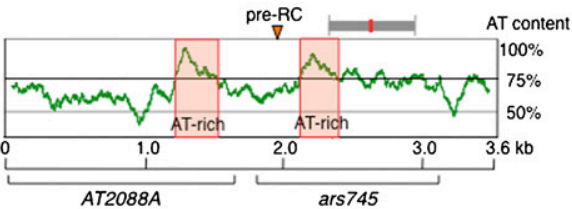

D

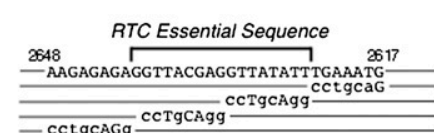

$E$

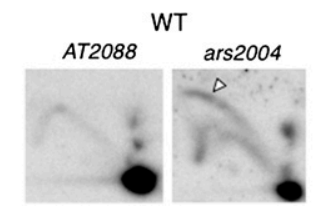

3.6
$\mathrm{~kb}$ Replication of ars2004 (ratio with/without insertion) \begin{tabular}{llllll}
0.2 & 0.4 & 0.6 & 0.8 & 1.0 & 1.2 \\
\hline
\end{tabular}
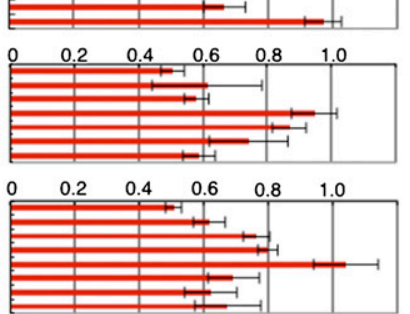

Replication of ars2004 (ratio with basesubstituted AT2088 / without AT2088)

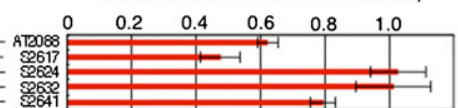

AT2088 210

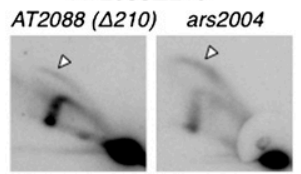

Figure 3. Identification of the essential sequence for RTC. (A) The structure of the ars2004 locus with the insertion of AT2088 fragment is presented schematically. $(B)$ Cells carrying the insertion of various portions of the AT2088 fragment (blue horizontal bars) next to the ars2004 locus were synchronously released from G2/M and labeled with BrdU in the presence of $\mathrm{HU}$ (10 $\mathrm{mM}$ ) for $120 \mathrm{~min}$ (top panel), $90 \mathrm{~min}$ (middle panel), or 80 min (bottom panel). Replication (percentage) of the ars2004 and that of the internal control origin AT2024 were determined as mean \pm SD from multiple qPCR measurements, as described in Figure 2. To compare the effects of the insertions on early replication of ars2004, relative replication of ars2004 to that of AT2024 was obtained, and the ratio of relative replication (ars2004/AT2024) with insertion to value without insertion was determined (red horizontal bars). The results of biologically independent experiments are presented in Supplemental Figure S3A. $(C)$ The profile of AT content of every $100-\mathrm{bp}$ window in a $3.6-\mathrm{kb}$ AT2088 region is presented together with two AT-rich regions $(>75 \%$, shaded rectangles), the pre-RC site (arrowhead) (Hayashi et al. 2007), and the locations of two ARS fragments, AT2088A (Supplemental Fig. S3B) and ars 745 (Maundrell et al. 1988). The regions sufficient (gray) and essential (red) for RTC are shown above the AT content profile. $(D)$ The 3.6-kb AT2088 fragment with base substitutions in the essential region from position 2617 to 2648 , as determined in $B$, was inserted next to the ars2004, and the effects of base substitutions on the repression of early replication of ars2004 were analyzed as described in $B$. The nucleotide sequence of the complementary strand of 2617-2648 is presented together with base substitutions (lowercase letters). The RTC essential sequence is indicated. $(E)$ Replication intermediates in the HU-treated wild-type (left panels) and AT20884210 (right panels) cells lacking the 210-bp (2527-2736) sequence were analyzed by 2D gel electrophoresis for ars2004 (EcoT22I fragment) and AT2088 (FbaI-NcoI fragment) at $90 \mathrm{~min}$ after G2/M release. Arrowheads indicate the bubble arcs.

ARS activity (Supplemental Fig. S3B), confirming that RTC is distinct from the origin activity.

We here examined whether the RTC controls replication timing at the original AT2088 locus. A 210-bp fragment containing the RTC essential sequence was deleted from the AT2088 locus (AT20884210), and replication initiation was analyzed by $2 \mathrm{D}$ gel electrophoresis. In contrast to a faint $\mathrm{Y}$ arc from the AT2088 locus in HUtreated wild-type cells, a bubble arc, indicative of the initiation, was generated along with a strong transition $\mathrm{Y}$ arc resulting from initiation from an asymmetrical position within the restriction fragment in AT20884210 (Fig. $3 \mathrm{E})$. These results indicate that the RTC is required for the control of replication timing at the native AT2088 locus.

\section{Telomeric repeats are essential for repression of internal late replication origins}

In fission yeast, telomeric repeats consist of GGTTAC followed by $\mathrm{A}_{0-1} \mathrm{C}_{0-1} \mathrm{G}_{0-6}$ variations (Sugawara 1989; Cooper et al. 1997; Hiraoka et al. 1998). We found that the RTC essential sequence contains two tandem copies of a telomeric repeat, with a substitution of $\mathrm{T}$ for the last $\mathrm{C}$ (Fig. 4B).
We examined whether the telomeric repeats are essential for the control of replication timing at the native AT2088 locus. Base substitution of the essential sequence (AT2088-S2632) promoted early replication specifically at AT2088 (Fig. 4B). Because the telomeric repeats in the RTC essential sequence contain an incomplete match to the consensus, we confirmed that two copies of the telomere consensus sequence GGGTTACAGGTTAC AGGG replaced functionally the incomplete sequence (Fig. 4B, AT2088-telF). Insertion of the consensus in the opposite direction also restored the RTC (Fig. 4B, AT2088telR), suggesting that the telomeric repeats act as a discrete element, such as the protein-binding site.

To explore the general role of telomeric repeats in the control of replication timing in fission yeast, we searched the fission yeast genome for two tandem telomeric sequences-GGTTAY-N (1-3) -GGTTAY, where Y represents pyrimidine-using the "fuzznuc" program (see the Materials and Methods). Such telomeric repeats are located at 26 loci, exclusively in the intergenic regions (Supplemental Tables S1, S2). Interestingly, 13 of them exist within the same intergenic spaces with late origins, which were identified in a previous genome-wide study 
A

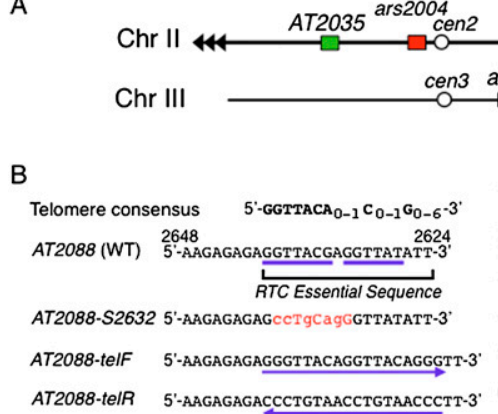

C

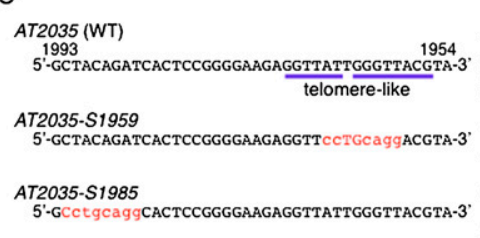

5'-GCctgcaggCACTCCGGGGAAGAGGTTATtGGGTTACGTA-3'

\section{D}

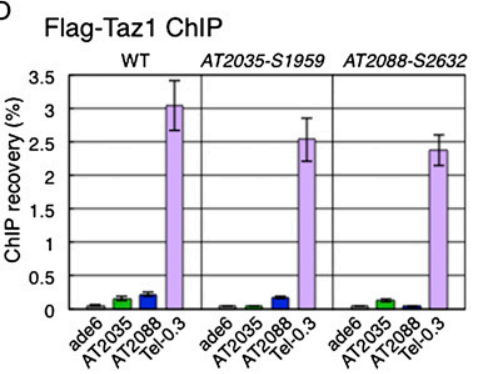

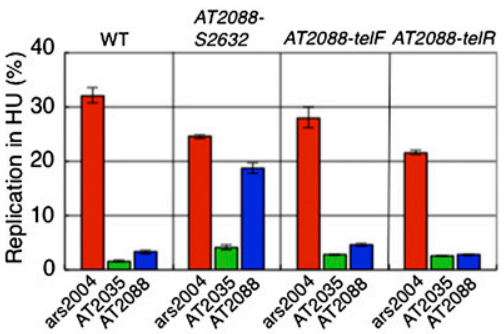
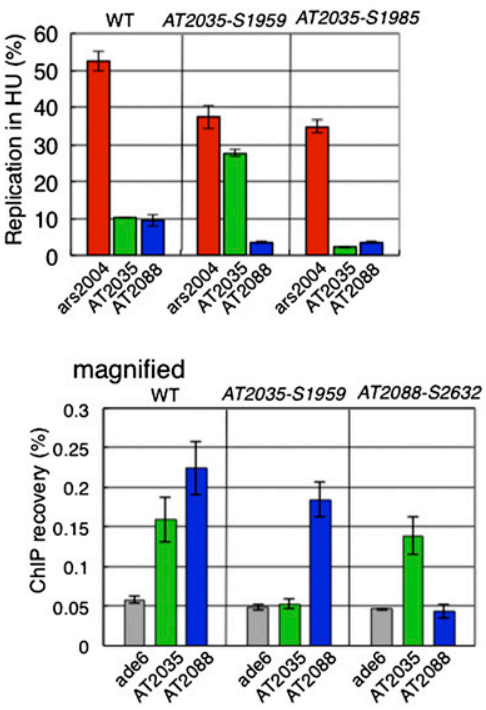

Figure 4. Telomeric repeats that recruit Tazl are essential for repression of late replication origins. $(A)$ The positions of AT2035 (green), AT2088 (blue), and Tel-0.3 (purple) on chromosome II and of ade6 (gray) on chromosome III are presented schematically. $(B$, left) The nucleotide sequences of the relevant regions of AT2088 (2624-2648, complementary strand), containing two copies of telomere-like repeats (blue underlining); AT2088-S2632, carrying base substitutions (red); and AT2088-telF and AT2088-telR,with two telomeric repeats (blue underlining) in either orientation, respectively, are presented together with the consensus sequence of fission yeast telomeres (Cooper et al. 1997). (Right) Replication (percentage) of the AT2088 (blue), ars2004 (red), and AT2035 (green) was measured in the HU-treated wild type and in strains carrying base substitutions in AT2088 at $120 \mathrm{~min}$ after G2/M release as described in Figure 2 . The mean \pm SD obtained from multiple measurements in qPCR is presented. The results of biologically independent experiments are shown in Supplemental Figure S4A. (C) Replication of the AT2035 was examined as described in $B$. The nucleotide sequences of the region (1954-1993, complementary strand) containing telomere-like repeats (blue underlining) and base substitutions (red) in AT2035-S1959 and AT2035-S1985 are presented. The results of biologically independent experiments are shown in Supplemental Figure S4B. $(D)$ Flag-Taz1 was immunoprecipitated from asynchronously cultured wild-type, AT2035-S1959, and AT2088-S2632 cells. Immunoprecipitation recovery of the chromosome locus against the total cellular DNA was measured by qPCR for ade ${ }^{+}$(gray), AT2035 (green), AT2088 (blue), and Tel-0.3 (purple). Bars indicate the mean of immunoprecipitation recovery (percentage) with standard error (SE) obtained from three biologically independent experiments. (Right) The results, except for those of Tel-0.3, are presented for a better comparison of Tazl localization at the internal sites.

(Hayashi et al. 2007). To investigate whether the telomeric repeats have a general role in RTC, we disrupted the one located near AT2035 on chromosome II (Fig. 4C). Base substitutions in the telomeric repeats caused robust early replication specifically at AT2035 (Fig. 4C, AT2035S1959). These results strongly suggest that the telomeric repeats present near internal late origins play a general role in RTC.

\section{Taz1 binds to the telomeric repeats near the internal} late origins

At the ends of chromosomes, Taz1, a fission yeast ortholog of human TRF1 and TRF2, binds to two tandem copies of the telomeric repeat (Cooper et al. 1997; Spink et al. 2000). To elucidate the role of telomeric repeats located near the internal late origins in the control of replication timing, we analyzed localization of 6Flag-tagged Tazl at the internal chromosome regions using a chromatin immunoprecipitation (ChIP) assay. ChIP recovery of DNA for ade6, AT2035, AT2088, and the telomere-proximal fragment (Tel-0.3) was determined by qPCR (Fig. 4D). In wild-type cells, the recovery of Tel-0.3 was $\sim 50$-fold higher than that of ade6 (Fig. 4D, left), indicating the accumulation of Taz1 at the telomeres. Taz1 was also localized at telomeric repeats near AT2035 and AT2088, as shown by recovery threefold and fourfold higher than that of ade6, respectively (Fig. 4D, right). The localization of Tazl at AT2035 was impaired by AT2035-S1959 base substitution, while the localization at $A T 2088$ was not affected (Fig. 4D, right). Moreover, AT2088-S2632 substitution abolished the Taz1 localization specifically at the AT2088 locus (Fig. 4D, right). These results demonstrate that the telomeric repeats near AT2035 and AT2088 recruit Taz1 to the corresponding loci.

Two copies of RTC essential sequence are sufficient for control of the replication timing of the nearby replication origin

Because telomeric repeats are essential for the timing control of AT2088 and AT2035 (Fig. 4B,C), we tested whether telomeric repeats are sufficient for control of late replication timing. To this end, two copies of the $25 \mathrm{bp}$ of the DNA fragment (2RTC25) containing the RTC essential sequence of $A T 2088$ were integrated next to the ars2004 locus, and the replication of the ars2004 in HUtreated cells was analyzed (Fig. 5A). A BrdU-incorporation 
Tazumi et al.

A

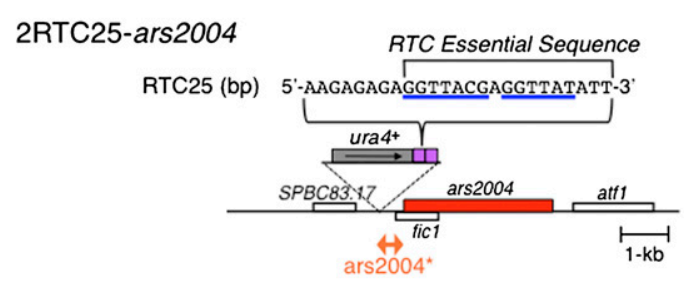

B

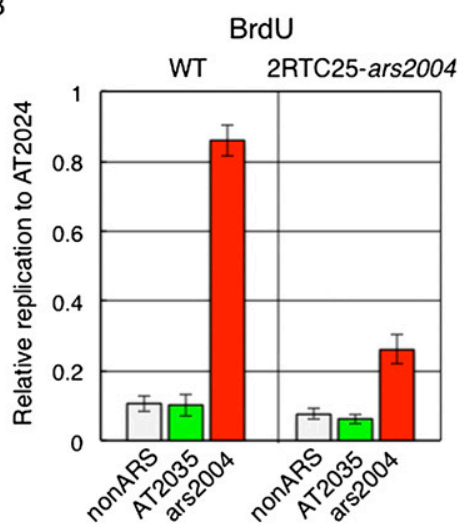

C
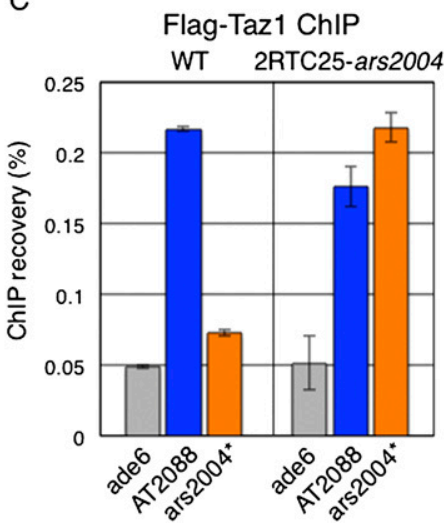

Figure 5. Two copies of the RTC essential sequence are sufficient for recruitment of Tazl and repression of the nearby early origin. (A) Two copies of the 25-bp fragment (RTC25) containing the RTC essential sequence (purple boxes), inserted next to the ars2004 locus, are presented schematically. A fragment (ars2004*) used for qPCR analysis is shown below the map. (B) cdc25-22 nmt1-TK ${ }^{+}$cells with (2RTC25-ars2004) or without (wild type [WT]) the insertion of 2RTC25 were released synchronously from G2/M and labeled with $\mathrm{BrdU}$ in the presence of $\mathrm{HU}(10 \mathrm{mM})$ for $120 \mathrm{~min}$. The ratios of the replicated population of non-ARS, AT2035, and ars2004 loci to that of the AT2024 locus were obtained as described in Figure 3B. $(C)$ Localization of Tazl at the inserted 2RTC25 was analyzed by ChIP assay. Flag-Tazl was immunoprecipitated from asynchronously cultured wild-type (WT) and 2RTC25ars2004 cells. Immunoprecipitation recovery of the chromosome loci against the total cellular DNA was measured by qPCR for adeb $^{+}$(gray), AT2088 (blue), and ars2004* (orange). The mean \pm SD obtained from multiple measurements in qPCR is presented. assay showed that the insertion of 2RTC25 decreased the replication of ars2004, indicating that the telomeric repeats are sufficient for the repression of early replication of the neighboring origin (Fig. 5B). Then, we analyzed whether the 2RTC25 recruited Tazl to the locus using a ChIP assay (Fig. 5C). In wild-type cells without insertion, Tazl was not localized at the locus near ars2004 (Fig. 5C, WT). In contrast, Tazl was localized to the locus in the 2RTC25-ars2004 cells (Fig. 5C, 2RTC25-ars2004). These results indicate that the telomeric repeats are sufficient for the recruitment of Tazl and for the timing control of a nearby replication origin.

\section{Taz1 plays an important role in the global control of replication timing}

We then asked whether Tazl is required for RTC at late replication origins. BrdU incorporation was determined in the wild-type and taz1D strains for AT2035 and AT2088, which are associated with telomeric repeats, as well as late origins AT2080 and ARS727, which are not associated with telomeric repeats, and the subtelomeric TAS59. In HU-treated wild-type cells, the early origin ars2004, but none of the late origins, replicated significantly (Fig. 6A). In contrast, in HU-treated taz1s cells, AT2035 and AT2088 showed robust replication to a level comparable with ars2004, whereas AT2080 or ARS727 did not replicate significantly (Fig. 6A). TAS59, which is located $\sim 20-\mathrm{kb}$ distant from the telomere, replicated more efficiently than ars2004 (Fig. 6A). These results indicate that Tazl is required for RTC of a subset of late origins that is associated with telomeric repeats.

To investigate the role of Tazl in the global control of replication timing, we analyzed the replication profile of all chromosomes. The BrdU-labeled DNA prepared from
HU-treated wild-type and taz1s cells was subjected to sequencing by oligonucleotide ligation and detection (SOLiD) (Fig. 6B; Supplemental Fig. S5). Comparison of the replication profiles in wild-type and taz1s cells revealed that among 156 late/dormant origins, 26 in chromosome arm regions and 46 in subtelomeres fired in the HU-treated taz1 $\Delta$ cells (Fig. 6D; Supplemental Fig. S5; Supplemental Table S1). Genome-wide analysis of Taz1 localization showed that Tazl was localized at 16 of 26 internally located late origins that fired in $\operatorname{taz} 1 \Delta$ (Fig. 6B,D; Supplemental Fig. S5; Supplemental Tables S1, S2). Notably, the entire subtelomeric region, spanning 50-100 $\mathrm{kb}$ from the ends of chromosomes I and II, including 46 previously identified late origins, replicated robustly in taz1s cells, although Taz1 was localized only in the vicinity of the telomere (Fig. 6C; Supplemental Fig. S5). These results suggest that Tazl plays an important role in the control of global replication timing in fission yeast.

\section{Taz1-mediated RTC prevents loading of Sld3 to late origins}

Because pre-RCs are formed at both early and late replication origins, we assumed the process of pre-RC activation to be regulated by the Taz1-mediated mechanism. To elucidate the step that is regulated, we analyzed the localizations of a pre-RC component (Mcm6) and Sld3, which is recruited to pre-RC in the most upstream step of the origin activation process in $S$ phase (Yabuuchi et al. 2006). Because Sld3 localization occurs only transiently under wild-type conditions, we employed the coldsensitive nda4-108/mcm5 mutation that allows Sld3 loading but prevents the initiation of replication (Yamada et al. 2004). In cdc25-22 nda4-108 sld3-Flag cells carrying the native AT2088 (wild type) released synchronously 
A

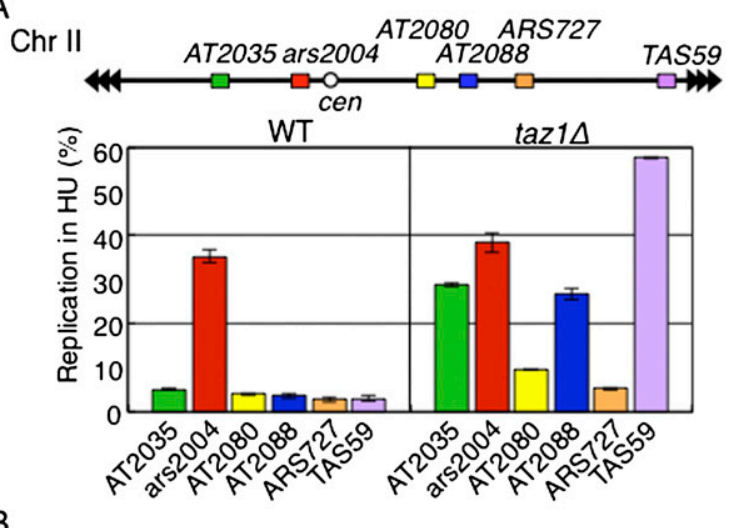

B
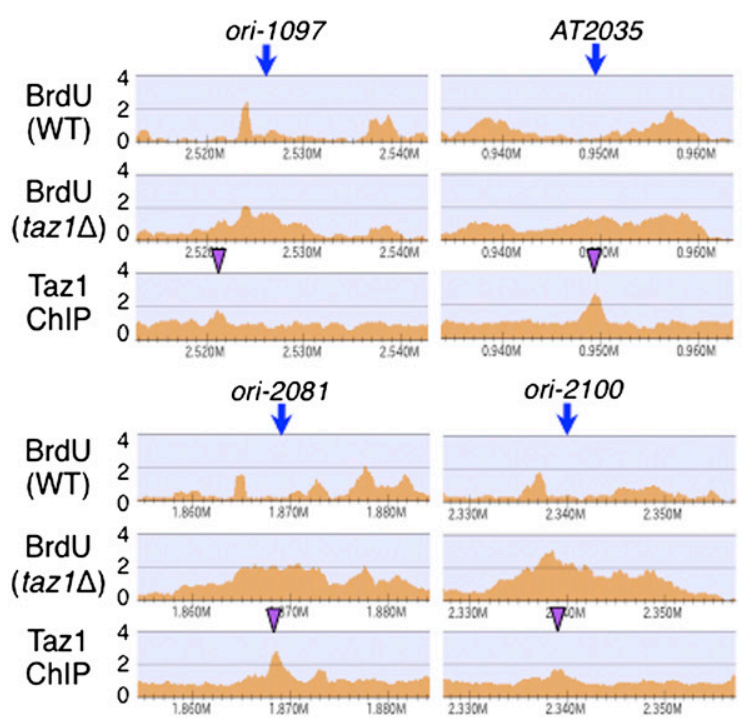

C
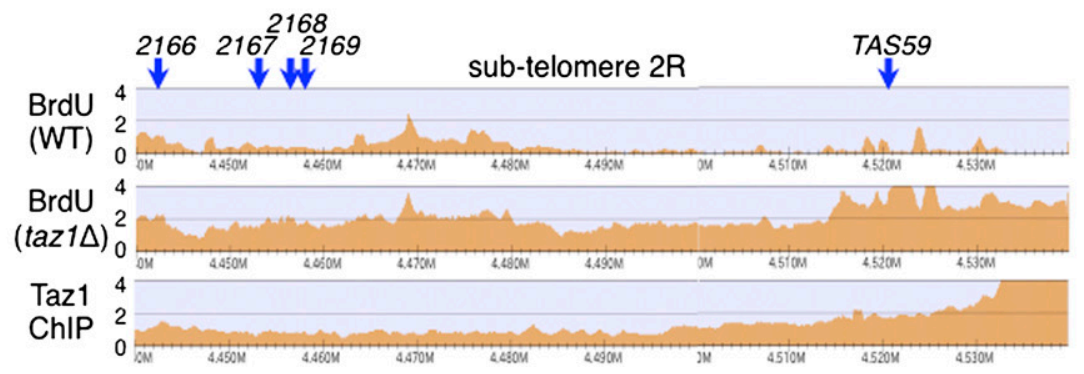

Figure 6. Tazl is required for repression of a subset of late replication origins. (A) Replication (percentage) of AT2035 (green), ars2004 (red), AT2088 (blue), ARS727 (orange), and TAS59 (purple) was determined in HU-treated wild-type and taz1A cells at 120 min after release from G2/M. The mean \pm SD obtained from multiple measurements in $\mathrm{qPCR}$ is presented. (B) BrdU incorporation profiles of six late origins associated with the telomeric repeats in wild type (top) and taz1s (middle) are presented together with profiles of Tazl-ChIP-seq (bottom). The sequence read from the HL density DNA prepared from a 120-min sample after G2/M release in HU-treated wild type (top) and taz1 (middle) was compared with the sequence read from the total cellular DNA of the respective cells, and the relevant enrichment was plotted over the chromosome region (Supplemental Fig. S5). (Bottom) Flag-Taz1 was immunoprecipitated as described in Figure 4D, and the relevant enrichment, the sequence read from ChIP in Flag-taz1 cells versus the sequence read from ChIP in the nontag strain, was plotted. The positions of late origins and telomeric repeats are shown by blue arrows and purple triangles, respectively. The results covering the entire genome are presented in Supplemental Figure S5 and summarized in Supplemental Table S1. (C) Replication profile of the subtelomere $2 \mathrm{R}$ is presented. Blue arrows show late origins. $(D)$ Comparison of late origins that are activated in taz1 $1 \Delta$. A Venn diagram shows 72 late origins activated in taz1 (purple) that contain 46 subtelomeric and 26 internal origins (white), including 16 internal origins where Tazl was localized (blue).

into S phase from G2/M, Mcm6 was efficiently localized at all the origins but not in the nonorigin (nonARS) region (Fig. 7A, left). Sld3 was preferentially enriched at the ars2004 but not at late origins or non-ARS (Fig. 7B, left). In AT2088-S2632 cells lacking the telomeric repeat near AT2088, localization of Sld3 increased specifically 
A

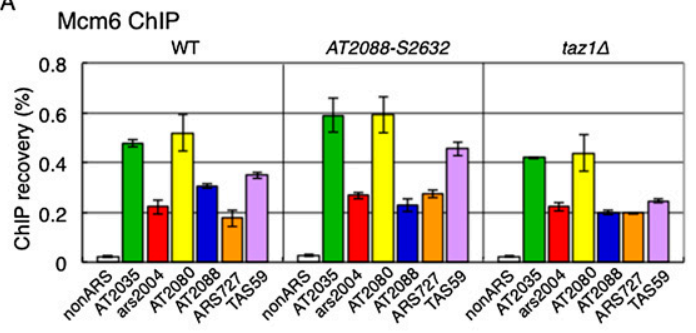

B

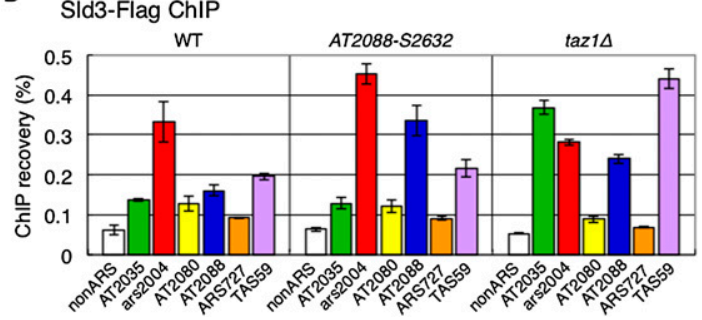

Figure 7. Recruitment of Sld 3 to a subset of late origins in taz1s. Localizations of Mcm6 $(A)$ and Sld3-5Flag $(B)$ at replication origins was analyzed by ChIP assay. Wild-type, AT2088S2632, and taz14 strains carrying the nda4-108/mcm5 and cdc25-22 mutations were synchronously released from the $\mathrm{G} 2 / \mathrm{M}$ boundary and incubated for $120 \mathrm{~min}$ at $20^{\circ} \mathrm{C}$, causing arrest before initiation of replication due to the nda4-108 mutation (Yamada et al. 2004). DNA of the indicated loci recovered by immunoprecipitation with Mcm6 or Sld3-5Flag was measured by qPCR for non-ARS (white), AT2035 (green), ars2004 (red), AT2080 (yellow), AT2088 (blue), ARS727 (orange), and TAS59 (purple). The mean \pm SD obtained from multiple measurements in qPCR is presented. The results of biologically independent experiments are shown in Supplemental Figure S6.

at $A T 2088$ (Fig. 7B, middle), whereas Mcm6 localizations were not significantly different from those in the wild type (Fig. 7A, middle). These results show that the telomeric repeat-dependent RTC activity represses Sld3 loading. We then examined whether Tazl impacted the localization of Mcm6 or Sld3. In the taz1s strain, localization of Sld3 increased specifically at AT2035, AT2088, and TAS59 but not at AT2080 or ARS727 (Fig. 7B, right), while Mcm6 was localized at the origins, similarly to those in wild type (Fig. 7A, right). These results show that Tazl-mediated control of replication timing does not affect Mcm6 localization but prevents Sld3 loading at specific late origins.

\section{Discussion}

A replication timing program that regulates individual replication origins to fire at a prefixed time during $S$ phase exists in all eukaryotes. In this study, we demonstrate that replication timing at a late origin is intrinsically determined by a proximal segment in fission yeast. The segment has the activity to repress initiation from nearby origins, even at ectopic sites, in early $\mathrm{S}$ phase, and two copies of the telomeric repeat are essential for this repression. A conserved telomere-binding protein, Tazl, binds to the internal telomeric repeats and plays an essential role in RTC. Genome-wide analysis revealed that about half of late origins are regulated by the Taz1mediated mechanism. Remarkably, almost all origins in subtelomeric regions $\sim 100 \mathrm{~kb}$ long were found to be regulated by Tazl. Our findings provide novel insights into the mechanism of RTC, especially how chromosome regions are distinctly regulated.

\section{Roles of Taz1 in the control of replication timing}

Our results demonstrate that two tandem copies of a telomeric repeat located in the vicinity of two late origins, AT2088 and AT2035, are essential for the control of replication timing at these origins. The results of ChIP analysis demonstrated that the telomeric repeats, which are the binding sites for a homodimeric Tazl (Cooper et al. 1997; Spink et al. 2000), indeed recruit Taz1 to the internal chromosome loci (Fig. 4). Moreover, Tazl is required for the timing control of about half of the late origins (72 among 156), including all of the subtelomeric origins (46 origins). Genome-wide analysis of Taz1 localization showed that Tazl binds to the vicinity of 16 among 26 internal late origins that are regulated in a Taz1-dependent manner (Fig. 6; Supplemental Fig. S5; Supplemental Table S1). Because 13 of the 16 origins are associated with telomeric repeats (including three that were manually found), Taz1 plays a role in the control of replication timing through binding to telomeric repeats near these origins. On the other hand, Tazl localization was not detected at 10 internal late origins that are activated in taz1s cells, leaving the possibility that Tazl can participate in the control from a distance through a certain mechanism. It is also remarkable that control of all of the subtelomeric late origins are dependent on Taz1, despite the absence of Taz1 localization at the individual origins except for in the vicinity of the telomeres (Supplemental Fig. S5; Supplemental Table S1; Kanoh et al. 2005). It is likely that Taz1 that binds to the telomere may play a role in the control of subtelomeric origins. Because the amount of telomere-bound Tazl varies depending on the length of the individual telomere, Tazl monitors the telomere length and impacts the timing of subtelomeric replication. This is consistent with the observations that shortened telomeres induce early firing of subtelomeric replication origins in budding yeast, in which Rap1 plays the role of Taz1 (Bianchi and Shore 2007; Shore and Bianchi 2009; Lian et al. 2011). The timing of replication fork passage through the telomere is important for the stable association of the telomerase and the elongation of the telomere (Dionne and Wellinger 1998; Marcand et al. 2000; Gallardo et al. 2011). Thus, Tazl is likely to play crucial roles in the feedback loop of replication-mediated maintenance of telomere length in addition to its role in the passage of the replication forks through the telomeres (Miller et al. 2006).

Two copies of the 25-bp RTC essential sequence containing four telomeric repeats are sufficient for late timing control of a nearby early origin (Fig. 5), suggesting the critical role of the telomeric sequence and binding of Tazl. In addition to the telomeric sequence, a flanking AAGAGAGA sequence has some importance for the 
RTC activity (Fig. 3D; data not shown). A similar purine cluster is found flanking the telomeric sequence near AT2035 (Fig. 4C). A purine-rich sequence may either stimulate Taz1 binding or recruit a factor involved in the repression of replication initiation. In addition, a region of $\sim 60 \mathrm{bp}(2557-2617)$ adjacent to the essential sequence contributes to origin repression to some extent (Fig. 3). Since the region is included in the scaffold/ matrix-associating regions (SAR/MARs) (Amati and Gasser 1990), association with the nuclear scaffold may restrict the access of replication factors or increase the local concentration of inhibitory factors.

What is the significance of Tazl-dependent RTC in the internal chromosome regions? About half of the telomeric repeat-associated late origins (nine among 13), including ori2081, ori2100, and AT2088/ori2114, are flanked by the genes that are silenced in normal growth conditions and induced under various stresses or during meiosis (Supplemental Table S2). Since gene expression at these loci might be regulated by the mechanisms involving specific transcription factors and histone modifications, control of replication timing may contribute to the maintenance or alteration of histone modifications. Although none of the Tazl-dependent internal late origins, except several subtelomeric origins in the vicinity to the telomeres, are activated in early premeiotic S phase (Heichinger et al. 2006), further investigations of relations between replication timing and gene expression will be needed. In metazoan, histone acetylation status is distinctly regulated during replication in the early and late $S$ phases, suggesting that a change in replication timing can play a role in the alteration of gene expression during development (Lande-Diner et al. 2009).

Recently, the observation that Rif1, another telomerebinding protein in fission yeast, is involved in the global control of replication timing has been reported (Hayano et al. 2012). Genome-wide analysis of replication in $\operatorname{taz} 1 \Delta$ as well as rif1 $\Delta$ revealed that all of the 72 late origins that fire in $\operatorname{taz} 1 \Delta$ are also activated in rif1s (Supplemental Figs. S5, S7; Supplemental Table S1). Moreover, 47 late origins that do not fire in taz1s, including AT2080 and ARS727/AT2013, are activated in rif1 $\Delta$ (Supplemental Figs. S5, S7; Supplemental Table S1). These observations suggest that there are at least Tazl-dependent and Tazl-independent pathways for timing control and both require Rif1. It is likely that Rif1 acts downstream from Taz1. It has been reported that the ARS727 fragment cloned on a plasmid forces late replication of a concurrently cloned early origin and that several copies of the late consensus sequence (LCS) A/G-G/T-G/T-GGGGGA-A/T are required for the repression (Yompakdee and Huberman 2004). The LCS might be involved in a Taz1-independent Rif1-dependent pathway (Hayano et al. 2012). At present, it is not known whether Taz1 recruits Rif1 to Tazl-dependent late origins such as AT2088. Further examination will be needed to understanding the mechanisms in which telomere-binding proteins Tazl and Rifl function to repress initiation of replication.
The Taz1-dependent pathway regulates DDKdependent Sld3 loading in the initiation of replication

In the Taz1-dependent control of replication timing described here, Tazl that binds in the vicinity of late origin impacts the process of initiation. The effects diminish by distance because an early origin $20 \mathrm{~kb}$ from the Taz1binding site is not inhibited (Supplemental Fig. S5). These characteristics suggest that a catalytic reaction or a semidiffusible factor may be involved in the interference with the process of replication initiation. The heterochromatin formation does not play a significant role because late replication of $A T 2088$ is maintained in the heterochromatin-deficient $s w i 6 \Delta$ or clr4s strain (A Tazumi, unpubl.). Checkpoint pathways, which are proficient in taz1s (Miller and Cooper 2003), may not play a major role in the Taz1-dependent timing control. Therefore, the Tazl-dependent regulation seems to involve a novel mechanism that affects the initiation process.

In this study, we showed that loading of Sld3 onto pre$\mathrm{RC}$ is prevented at late origins by a Tazl-mediated control of replication timing. In fission yeast, loading of Sld3 onto replication origins is dependent on pre-RC and DDK and is a prerequisite for CDK-dependent assembly of other replication factors (Yabuuchi et al. 2006; Fukuura et al. 2011). A recent in vitro study in budding yeast has shown that the origin association of Sld3 is dependent on DDK and is distinct from those that require CDK (Heller et al. 2011). Moreover, an in vivo study in budding yeast suggests that the origin association of low-abundance Sld3, Sld7, and Cdc45, which is dependent on DDK, is crucial for the temporal order of origin firing (Tanaka et al. 2011). The following evidence in fission yeast further emphasizes the importance of DDK recruitment in RTC: Swi6/ HP1-dependent recruitment of Dfp1, a regulatory subunit of DDK, stimulates early activation of origins in pericentromeric heterochromatin and the silent mat locus (Hayashi et al. 2009). Furthermore, tethering of Dfp1 to the late origin ARS727, which is Taz1-independent and Rif1-dependent, causes early firing of the origin (Patel et al. 2008). Because DDK preferentially phosphorylates subunits of the chromatin-bound Mcm2-7 complex (Francis et al. 2009), both Taz1-dependent and -independent regulation may interfere with the access of DDK or the phosphorylation of the chromatin-bound Mcm2-7 complex by DDK (Francis et al. 2009).

Although recruitment of DDK and loading of Sld3 occur in S phase, a process that occurs in G1 phase may impact the reactions. Correlation of the origin association timing of the ORC and MCM in G1 phase with initiation timing has been reported in fission yeast $\mathrm{Wu}$ and Nurse 2009). In mammalian cells, it has been proposed that a replication timing program is established during a discrete window of time in G1 phase, the TDP (Dimitrova and Gilbert 1999). It is possible that an early event such as chromatin modifications around individual replication origins could affect the recruitment of DDK and subsequent loading of replication factors at the origins in S phase. 
Our results show that fission yeast uses a set of telomeric sequences and the binding protein Tazl for regulation of replication timing. Recent studies revealed novel genome-wide functions of telomere-binding proteins in mammals. Mouse Rap1 binds to two tandem copies of the telomere repeat outside telomeric regions and regulates the expression of the genes located in the vicinity of the repeats (Martinez et al. 2010). In addition, mammalian Rap1 has also been identified as a regulator of the NF- $\mathrm{BB}$ signaling pathway that mediates the transcriptional responses to various stresses (Teo et al. 2010). Since telomeric sequences and telomere-binding proteins are highly conserved among eukaryotes, it would be interesting to investigate whether they could be involved in the regulation of DNA replication in other organisms.

\section{Materials and methods}

\section{Strains and media}

Strains and media used in this study are described in the Supplemental Material and are listed in Supplemental Table S3.

\section{BrdU incorporation and replication timing analysis}

Fission yeast strains carrying Pnmt1-TK cdc25-22 were cultured in EMM medium lacking thiamine to induce transcription of the TK gene for $18 \mathrm{~h}$ at $25^{\circ} \mathrm{C}$ to $5 \times 10^{6}$ cells per milliliter. Cells arrested at the G2/M boundary by cdc25-22 mutation for $3 \mathrm{~h}$ at $36^{\circ} \mathrm{C}$ were released at $25^{\circ} \mathrm{C}$ and cultured for the indicated periods in the presence of $200 \mu \mathrm{M}$ BrdU. The preparation of cellular DNA and separation of BrdU-labeled DNA by $\mathrm{CsCl}$ density gradient centrifugation were carried out as described previously (Hayashi et al. 2007). For replication kinetics analysis, the amount of DNA in the HL and LL densities was determined by real-time PCR using the primer sets listed in Supplemental Table S4. Replication (percentage) at each time point was calculated with the equation $100 \times(1 / 2 \mathrm{HL}) /(1 / 2 \mathrm{HL}+\mathrm{LL})$. To determine replication (percentage) of specific loci in early $\mathrm{S}$ phase, $10 \mathrm{mM} \mathrm{HU}$ was added upon release from the G2/M block.

\section{ChIP}

ChIP assay was performed essentially as described previously (Hayashi et al. 2009), except for the following modifications. For Tazl ChIP, $\sim 2.5 \times 10^{8}$ Flag-taz1 cells grown at $30^{\circ} \mathrm{C}$ were disrupted with glass beads in $0.4 \mathrm{~mL}$ of lysis buffer $(50 \mathrm{mM}$ Tris- $\mathrm{HCl}$ at $\mathrm{pH} 8.0,280 \mathrm{mM} \mathrm{NaCl}, 1 \mathrm{mM}$ EDTA, $1 \%$ Triton $\mathrm{X}-100,0.1 \%$ sodium deoxycholate, $1 \mathrm{mM}$ phenylmethylsulfonyl fluoride, supplemented with proteinase inhibitor cocktail [Sigma-Aldrich]). The soluble extracts, diluted with lysis buffer to adjust the Triton X-100 to $0.3 \%$, were used for immunoprecipitation with magnetic beads (Dynal) conjugated with mouse monoclonal anti-Flag M2 (1:400; Sigma-Aldrich). For Mcm6 and Sld3 ChIP, cdc25-22 sld3-Flag nda4-108/mcm5 cells grown at $28^{\circ} \mathrm{C}$ were synchronized at the G2/M boundary for $3 \mathrm{~h}$ at $36^{\circ} \mathrm{C}$ and released at $20^{\circ} \mathrm{C}$, which is the restrictive temperature for the nda4-108 (Yamada et al. 2004). Cell extracts were used for ChIP assays with mouse anti-Flag and rabbit anti-Mcm6 antibodies. DNA prepared from whole-cell extracts or immunoprecipitated fractions was quantified by qPCR using SYBR Green I in the 7300 PCR system (Applied Biosystems).

\section{Nucleotide pattern search}

For the search of two copies of the telomere-like sequence, the pattern of GGTTAYN(1,3)GGTTAY was searched using the EMBOS "fuzznuc" program, which was obtained at http:// emboss.sourceforge.net/apps/release/6.3/emboss/apps/fuzznuc. html.

\section{SOLiD analysis}

BrdU-labeled DNA prepared at 120 min after G2/M release from HU-treated wild-type, $\operatorname{taz} 1 \Delta$, and rif1 $1 \Delta$ strains and separated by $\mathrm{CsCl}$ density gradient centrifugation and those obtained by FlagTazl immunoprecipitation were used for SOLiD deep-sequencing analysis, which was performed according to the manufacturer's standard protocol (Applied Biosystems).

\section{Accession numbers}

Sequence data have been deposited to the Sequence Read Archive (http://www.ncbi.nlm.nih.gov/Traces/sra) with the accession number SRP011412.

\section{Acknowledgments}

We thank Drs. Junko Kanoh and Fuyuki Ishikawa for providing plasmids and strains, Dr. Teruo Yasunaga for searching nucleotide sequence patterns, Dr. Julia P. Cooper for discussion, and Dr. Makoto Hayashi for critical reading of the manuscript and providing materials. This study was supported by a Grant-in-Aid from the Ministry of Education, Science, Technology, Sports, and Culture of Japan to H.M., and by a Grant-in-Aid for JSPS Fellows to A.T.

\section{References}

Amati B, Gasser SM. 1990. Drosophila scaffold-attached regions bind nuclear scaffolds and can function as ARS elements in both budding and fission yeasts. Mol Cell Biol 10: 5442-5454.

Bell SP, Dutta A. 2002. DNA replication in eukaryotic cells. Annu Rev Biochem 71: 333-374.

Bianchi A, Shore D. 2007. Early replication of short telomeres in budding yeast. Cell 128: 1051-1062.

Cooper JP, Nimmo ER, Allshire RC, Cech TR. 1997. Regulation of telomere length and function by a Myb-domain protein in fission yeast. Nature 385: 744-747.

Dimitrova DS, Gilbert DM. 1999. The spatial position and replication timing of chromosomal domains are both established in early G1 phase. Mol Cell 4: 983-993.

Dionne I, Wellinger RJ. 1998. Processing of telomeric DNA ends requires the passage of a replication fork. Nucleic Acids Res 26: $5365-5371$.

Ferguson BM, Fangman WL. 1992. A position effect on the time of replication origin activation in yeast. Cell 68: 333-339.

Francis LI, Randell JC, Takara TJ, Uchima L, Bell SP. 2009. Incorporation into the prereplicative complex activates the Mcm2-7 helicase for Cdc7-Dbf4 phosphorylation. Genes Dev 23: 643-654.

Friedman KL, Diller JD, Ferguson BM, Nyland SV, Brewer BJ, Fangman WL. 1996. Multiple determinants controlling activation of yeast replication origins late in S phase. Genes Dev 10: $1595-1607$.

Fukuura M, Nagao K, Obuse C, Takahashi TS, Nakagawa T, Masukata H. 2011. CDK promotes interactions of Sld3 and Drc1 with Cut5 for initiation of DNA replication in fission yeast. Mol Biol Cell 22: 2620-2633. 
Gallardo F, Laterreur N, Cusanelli E, Ouenzar F, Querido E, Wellinger RJ, Chartrand P. 2011. Live cell imaging of telomerase RNA dynamics reveals cell cycle-dependent clustering of telomerase at elongating telomeres. Mol Cell 44: 819-827.

Gilbert DM, Takebayashi SI, Ryba T, Lu J, Pope BD, Wilson KA, Hiratani I. 2010. Space and time in the nucleus: Developmental control of replication timing and chromosome architecture. Cold Spring Harb Symp Quant Biol 75: 143-153.

Hayano M, Kanoh Y, Matsumoto S, Renard-Guillet C, Shirahige K, Masai H. 2012. Rif1 is a global regulator of timing of replication origin firing in fission yeast. Genes Dev 26: 137-150.

Hayashi M, Katou Y, Itoh T, Tazumi A, Yamada Y, Takahashi T, Nakagawa T, Shirahige K, Masukata H. 2007. Genome-wide localization of pre-RC sites and identification of replication origins in fission yeast. EMBO J 26: 1327-1339.

Hayashi MT, Takahashi TS, Nakagawa T, Nakayama J, Masukata H. 2009. The heterochromatin protein Swi6/HP1 activates replication origins at the pericentromeric region and silent mating-type locus. Nat Cell Biol 11: 357-362.

Heichinger C, Penkett CJ, Bahler J, Nurse P. 2006. Genome-wide characterization of fission yeast DNA replication origins. EMBO J 25: 5171-5179.

Heller RC, Kang S, Lam WM, Chen S, Chan CS, Bell SP. 2011. Eukaryotic origin-dependent DNA replication in vitro reveals sequential action of DDK and S-CDK kinases. Cell 146: 80-91.

Hiraoka Y, Henderson E, Blackburn EH. 1998. Not so peculiar: Fission yeast telomere repeats. Trends Biochem Sci 23: 126. doi: 10.1016/S0968-0004(98)01176-1.

Hiratani I, Gilbert DM. 2009. Replication timing as an epigenetic mark. Epigenetics 4: 93-97.

Hiratani I, Leskovar A, Gilbert DM. 2004. Differentiation-induced replication-timing changes are restricted to AT-rich/ long interspersed nuclear element (LINE)-rich isochores. Proc Natl Acad Sci 101: 16861-16866.

Hiratani I, Ryba T, Itoh M, Yokochi T, Schwaiger M, Chang CW, Lyou Y, Townes TM, Schubeler D, Gilbert DM. 2008. Global reorganization of replication domains during embryonic stem cell differentiation. PLoS Biol 6: e245. doi: 10.1371/ journal.pbio.0060245.

Kanoh J, Ishikawa F. 2001. spRap1 and spRif1, recruited to telomeres by Taz1, are essential for telomere function in fission yeast. Curr Biol 11: 1624-1630.

Kanoh J, Sadaie M, Urano T, Ishikawa F. 2005. Telomere binding protein Tazl establishes Swi6 heterochromatin independently of RNAi at telomeres. Curr Biol 15: 1808-1819.

Kim SM, Dubey DD, Huberman JA. 2003. Early-replicating heterochromatin. Genes Dev 17: 330-335.

Labib K. 2010. How do Cdc7 and cyclin-dependent kinases trigger the initiation of chromosome replication in eukaryotic cells? Genes Dev 24: 1208-1219.

Lande-Diner L, Zhang J, Cedar H. 2009. Shifts in replication timing actively affect histone acetylation during nucleosome reassembly. Mol Cell 34: 767-774.

Lian HY, Robertson ED, Hiraga S, Alvino GM, Collingwood D, McCune HI, Sridhar A, Brewer BJ, Raghuraman MK, Donaldson $\mathrm{AD}$. 2011. The effect of $\mathrm{Ku}$ on telomere replication time is mediated by telomere length but is independent of histone tail acetylation. Mol Biol Cell 22: 1753-1765.

MacAlpine DM, Bell SP. 2005. A genomic view of eukaryotic DNA replication. Chromosome Res 13: 309-326.

MacAlpine DM, Rodriguez HK, Bell SP. 2004. Coordination of replication and transcription along a Drosophila chromosome. Genes Dev 18: 3094-3105.

Marcand S, Brevet V, Mann C, Gilson E. 2000. Cell cycle restriction of telomere elongation. Curr Biol 10: 487-490.
Martinez P, Thanasoula M, Carlos AR, Gomez-Lopez G, Tejera AM, Schoeftner S, Dominguez O, Pisano DG, Tarsounas M, Blasco MA. 2010. Mammalian Rapl controls telomere function and gene expression through binding to telomeric and extratelomeric sites. Nat Cell Biol 12: 768-780.

Maundrell K, Hutchison A, Shall S. 1988. Sequence analysis of ARS elements in fission yeast. EMBO J 7: 2203-2209.

Miller KM, Cooper JP. 2003. The telomere protein Tazl is required to prevent and repair genomic DNA breaks. Mol Cell 11: 303-313.

Miller KM, Rog O, Cooper JP. 2006. Semi-conservative DNA replication through telomeres requires Tazl. Nature 440: 824-828.

Okuno Y, Satoh H, Sekiguchi M, Masukata H. 1999. Clustered adenine/thymine stretches are essential for function of a fission yeast replication origin. Mol Cell Biol 19: 6699-6709.

Patel PK, Kommajosyula N, Rosebrock A, Bensimon A, Leatherwood J, Bechhoefer J, Rhind N. 2008. The Hsk1(Cdc7) replication kinase regulates origin efficiency. Mol Biol Cell 19: 5550-5558.

Raghuraman MK, Winzeler EA, Collingwood D, Hunt S, Wodicka L, Conway A, Lockhart DJ, Davis RW, Brewer BJ, Fangman WL. 2001. Replication dynamics of the yeast genome. Science 294: 115-121.

Remus D, Diffley JF. 2009. Eukaryotic DNA replication control: Lock and load, then fire. Curr Opin Cell Biol 21: 771-777.

Santocanale C, Diffley JF. 1998. A Mec1- and Rad53-dependent checkpoint controls late-firing origins of DNA replication. Nature 395: 615-618.

Santocanale C, Sharma K, Diffley JF. 1999. Activation of dormant origins of DNA replication in budding yeast. Genes Dev 13: 2360-2364.

Segurado M, de Luis A, Antequera F. 2003. Genome-wide distribution of DNA replication origins at A+T-rich islands in Schizosaccharomyces pombe. EMBO Rep 4: 1048-1053.

Sharma K, Weinberger M, Huberman JA. 2001. Roles for internal and flanking sequences in regulating the activity of mating-type-silencer-associated replication origins in Saccharomyces cerevisiae. Genetics 159: 35-45.

Shore D, Bianchi A. 2009. Telomere length regulation: Coupling DNA end processing to feedback regulation of telomerase. EMBO J 28: 2309-2322.

Spink KG, Evans RJ, Chambers A. 2000. Sequence-specific binding of Tazlp dimers to fission yeast telomeric DNA. Nucleic Acids Res 28: 527-533.

Sugawara N. 1989. "DNA sequences at the telomeres of fission yeast $S$. pombe." $\mathrm{PhD}$ thesis, Harvard University, Cambridge, MA.

Takahashi T, Ohara E, Nishitani H, Masukata H. 2003. Multiple ORC-binding sites are required for efficient MCM loading and origin firing in fission yeast. $E M B O J$ 22: 964-974.

Tanaka S, Araki H. 2010. Regulation of the initiation step of DNA replication by cyclin-dependent kinases. Chromosoma 119: $565-574$.

Tanaka S, Nakato R, Katou Y, Shirahige K, Araki H. 2011. Origin association of sld3, sld7, and cdc45 proteins is a key step for determination of origin-firing timing. Curr Biol 21: 2055-2063.

Teo H, Ghosh S, Luesch H, Ghosh A, Wong ET, Malik N, Orth A, de Jesus P, Perry AS, Oliver JD, et al. 2010. Telomereindependent Rap1 is an IKK adaptor and regulates NF-кBdependent gene expression. Nat Cell Biol 12: 758-767.

Wu PY, Nurse P. 2009. Establishing the program of origin firing during $S$ phase in fission Yeast. Cell 136: 852-864.

Wyrick JJ, Aparicio JG, Chen T, Barnett JD, Jennings EG, Young RA, Bell SP, Aparicio OM. 2001. Genome-wide distribution 
Tazumi et al.

of ORC and MCM proteins in S. cerevisiae: High-resolution mapping of replication origins. Science 294: 2357-2360.

Yabuuchi H, Yamada Y, Uchida T, Sunathvanichkul T, Nakagawa

T, Masukata H. 2006. Ordered assembly of Sld3, GINS and Cdc45 is distinctly regulated by DDK and CDK for activation of replication origins. EMBO J 25: 4663-4674.

Yamada Y, Nakagawa T, Masukata H. 2004. A novel intermediate in initiation complex assembly for fission yeast DNA replication. Mol Biol Cell 15: 3740-3750.

Yompakdee C, Huberman JA. 2004. Enforcement of late replication origin firing by clusters of short G-rich DNA sequences. J Biol Chem 279: 42337-42344. 


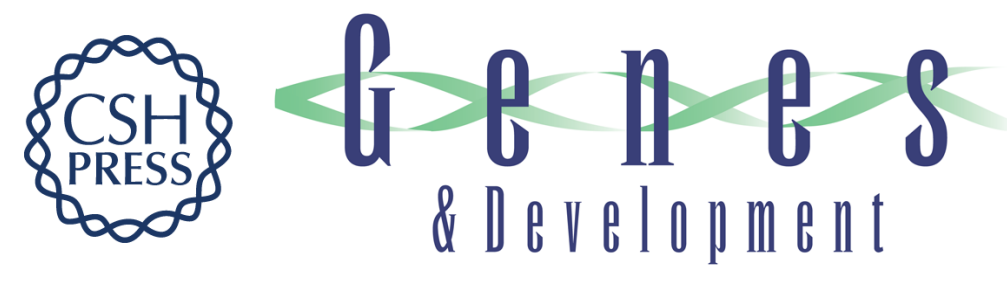

\section{Telomere-binding protein Taz1 controls global replication timing through its localization near late replication origins in fission yeast}

Atsutoshi Tazumi, Masayoshi Fukuura, Ryuichiro Nakato, et al.

Genes Dev. 2012, 26:

Access the most recent version at doi:10.1101/gad.194282.112

Supplemental http://genesdev.cshlp.org/content/suppl/2012/09/06/26.18.2050.DC1
Material

References This article cites 53 articles, 19 of which can be accessed free at:

http://genesdev.cshlp.org/content/26/18/2050.full.html\#ref-list-1

License

Email Alerting

Receive free email alerts when new articles cite this article - sign up in the box at the top

Service

right corner of the article or click here.

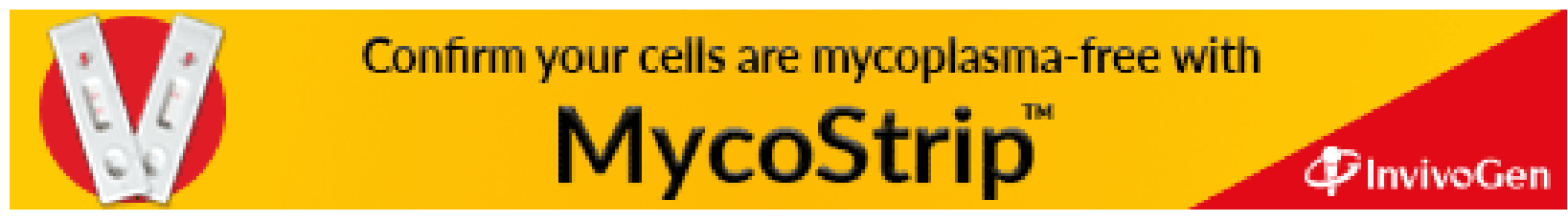

